\title{
Laparoscopic Surgery for Advanced Gastric Cancer: Current Status and Future Perspectives
}

\author{
Ichiro Uyama, Koichi Suda, and Seiji Satoh \\ Division of Upper Gastrointestinal Tract, Department of Surgery, Fujita Health University, Aichi, Japan
}

\begin{abstract}
Laparoscopic gastrectomy has been widely accepted especially in patients with early-stage gastric cancer. However, the safety and oncologic validity of laparoscopic gastrectomy for advanced gastric cancer are still being debated. Since the late 90s', we have been engaged in developing a stable and robust methodology of laparoscopic radical gastrectomy for advanced gastric cancer, and have established laparoscopic distinctive technique for suprapancreatic lymph node dissection, namely the outermost layer-oriented medial approach. In this article, We present the development history of this method, and current status and future perspectives of laparoscopic gastrectomy for advanced gastric cancer based on our experience and a review of the literature.
\end{abstract}

Key Words: Laparoscopy; Gastrectomy; Stomach neoplasms; Robotics; Anastomosis, surgical

\section{Introduction}

Gastric cancer is the fourth most common cancer and the second leading cause of cancer-related death worldwide. ${ }^{1}$ Surgical resection remains the only curative treatment option, and regional lymphadenectomy is recommended as part of radical gastrectomy. ${ }^{2}$ Laparoscopic gastrectomy (LG) is an emerging surgical approach which offers significant advantages on short-term outcomes when compared with open surgical procedures for patients with gastric cancer. $^{3-6}$ Although laparoscopic surgery is currently being regarded as the treatment of choice for early gastric cancer (EGC), the indications and outcomes of LG for advanced gastric cancer (AGC) remain controversial due to its technical difficulties and the lack of long-term results. This article provides a history of LG and gives

Correspondence to: Ichiro Uyama

Division of Upper Gastrointestinal, Department of Surgery, Fujita Health University, 1-98 Dengakugakubo, Kutsukake, Toyoake, Aichi, 470-1192 Japan

Tel: +81-562-93-9254, Fax: +81-562-93-9011

E-mail: iuyama@fujita-hu.ac.jp

Received February 19, 2013

Revised March 6, 2013

Accepted March 6, 2013 updated information on LG for AGC based on our experience along with a review of the literature.

\section{Body}

\section{Standard treatment for AGC}

The primary goal of surgery is to accomplish a complete resection with negative margins (R0 resection). ${ }^{3}$ The extent of lymph node dissection remains controversial. ${ }^{3}$

\section{1) In Japan}

The results of treatment for gastric cancer in Japan have improved markedly as a result of early detection and extensive radical surgery. ${ }^{8}$ In the 1960s, Japanese surgeons first introduced the extended lymphadenectomy procedure, known today as D2 dissection, which requires systematic dissection of lymph nodes in the 1st tier (perigastric) and the 2nd tier (along the celiac artery and its branches). ${ }^{2}$ Early studies have demonstrated that the 5 -year survival of patients with positive lymph node metastasis including the 2nd tier nodes ranged from 30 to $40 \%$ after D2 dissection. ${ }^{2}$ Since the 1980s, even more patients have undergone radical extended lymphadenectomy, namely, extended para-aortic 
Uyama I, et al

(D3) dissection, in many high-volume centers; however, a multiinstitutional, randomized, controlled trial (RCT) conducted by the Japan Clinical Oncology Group (JCOG9501) showed no survival benefit of D3 dissection compared to D2 at least in curable gastric cancer. $^{29,10}$ Thus, to date, the Japanese Gastric Cancer Association (JGCA) recommends that non-early, potentially curable gastric cancers should be treated by standard gastrectomy, which is the principle surgical procedure performed with curative intent, defined as resection of no less than two-thirds of the stomach with D2 lymph node dissection. ${ }^{9,11}$

\section{2) In the West}

There have been two major RCTs comparing D1 vs. D2 gastretomy in Europe. One is the Dutch Gastric Cancer Group Trial (Dutch trial), ${ }^{12,13}$ and the other is the British Cooperative trial conducted by the Medical Research Council (MRC trial). ${ }^{14}$ Initially, both trials not only failed to demonstrate the survival benefit of D2 dissection but also showed a significant association between D2 dissection and increased postoperative morbidity and mortality, suggesting that the technical aspects of D2 dissection require a significant degree of training and expertise. ${ }^{12,14}$ However, after a median follow-up of 15 years in the Dutch trial, D2 dissection was finally associated with lower loco-regional recurrence and gastriccancer related deaths than D1 dissection. ${ }^{13}$ Thus, in the West, the panel recommends that gastric cancer surgery should be performed by experienced surgeons in high volume cancer centers and should include removal of perigastric lymph nodes (D1) and those along the named vessels of the celiac axis (D2), with a goal of examining 15 or greater lymph nodes. ${ }^{3}$ In addition, both of these trials showed that splenectomy and pancreatectomy performed along with D2 dissection significantly increased the mortality and morbidity, thus modified D2 lymohadenectomy (without pancreatecctomy and splenectomy). ${ }^{3,12-14}$

\section{LG for $A G C$ at our institute}

\section{1) Development history}

Laparoscopic surgery was launched in the early 1990s in our country. ${ }^{15}$ At that time, most laparoscopic surgeons applied laparoscopic surgery, through its minimally invasive nature, to less extended surgeries. ${ }^{16}$ However, we assumed from the beginning that laparoscopic surgery should be allowed for meticulous dissection using a high quality of laparoscopic view with magnified visualization, even though there were a couple of limitations to laparoscopic surgery such as two-dimentional imaging with poor depth perception, long-handled forceps with a limited degree of freedom, shaking of the surgeon's hand, limited tactile sensation, and uncertain contribution to long-term survival. ${ }^{17}$ In 1995, we introduced laparoscopic assistance into moderate to advanced gastrointestinal and hepatobiliary surgery in combination with a proximal-to-distal and medial-to-lateral approach to overcome such limitations, and reported technical aspects of laparoscopic distal gastrectomy (LDG), ${ }^{18,19}$ proximal gastrectomy, ${ }^{20}$ pancreaticoduodenectomy, ${ }^{21}$ and hepatectomy.

In the late 90's, D2 but not D3 dissection turned to be recognized as a sufficient extent of lymph node dissection for AGC in Japan. ${ }^{8}$ Based on our prior experience in open gastrectomy (OG) with superextended lymphadenectomy ${ }^{23}$ as well as that in LG, it seemed to us that laparoscopic D2 dissection, which seemed like a "less extensive" surgery than D3 dissection, could be adequately achieved using a laparoscope. Therefore, we developed new techniques for laparoscopic distal and total gastrectomy with D2 dissection for $\mathrm{AGC}^{24,25}$ which were published for the first time in the world.

\section{2) Surgical devices}

To perform meticulous dissection laparoscopically, novel forceps and hemostats specialized for advanced laparoscopic surgery are required. In cooperation with Olympus Medical Systems Corp. (Tokyo, Japan), we developed Finger type Maryland Dissection forceps "WA64300A" (Fig. 1a), Left-hand type Grasping forceps

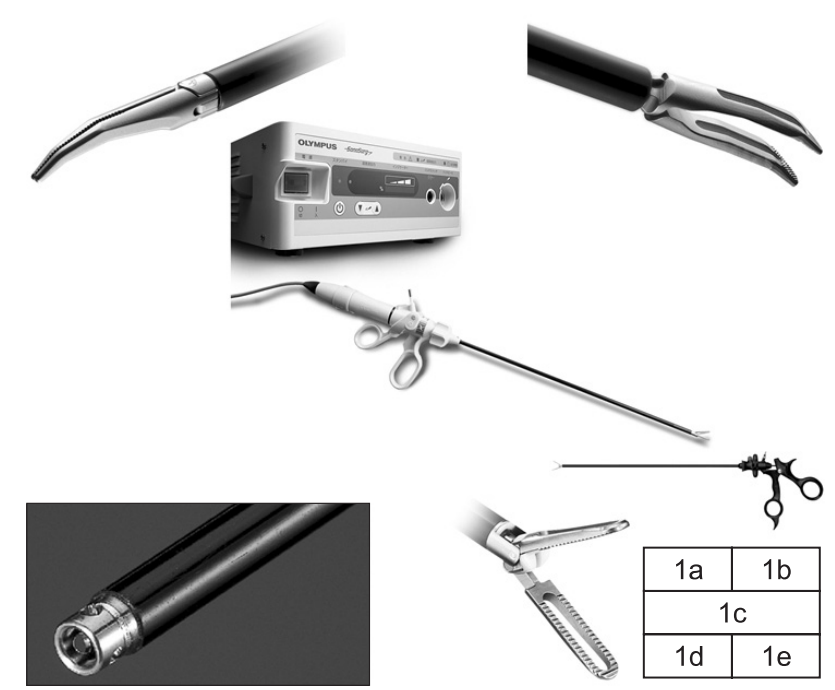

Fig. 1. Forceps and hemostats specialized for advanced laparoscopic surgery. 1a: Finger type Maryland Dissection forceps "WA64300A"; 1b: Left-hand type Grasping forceps "WA64360A"; 1c: Ultrasonic Surgical System "SonoSurg X"; 1d: Suction and irrigation tube with button electrode "WA51138A+WA51172S"; 1e: Johann type Bipolar Grasping forceps "WA64120C". 
Laparoscopic Radical Gastrectomy

"WA64360A" (Fig. 1b), Ultrasonic Surgical System "SonoSurg X” (Fig. 1c), Suction and irrigation tube with the button electrode "WA51138A+WA51172S" (Fig. 1d), and Johann type Bipolar Grasping forceps "WA64120C" (Fig. 1e), with a focus on the shape of the tip.

\section{3) Outermost layer-oriented medial approach}

According to the guidelines issued by JGCA, D2 dissection entails removal of lymph nodes in the suprapancreatic area in distal and total gastrectomy and at the splenic hilum in total gastrectomy. ${ }^{11}$ Dissection of these areas is technically demanding due to the serious risk of bleeding and/or bile and pancreatic leakage from a major vessel or organ injury. ${ }^{7}$ To improve the safety, efficacy, and reproducibility of suprapancreatic nodal dissection, we developed our original methodology called outermost layeroriented medial approach. ${ }^{17,26}$ In this approach, the layer between the autonomic nerve sheath of the major arteries and adipose tissue bearing lymphatic tissue is dissected (Fig. 2). ${ }^{17,26}$ We named this layer as the outermost layer of the autonomic nerve. ${ }^{17}$ To identify this layer throughout the dissection process, we developed an original surgical approach consisting of the following three steps: (1) medial dissection of the left gastric artery; (2) right suprapancreatic dissection; and (3) left suprapancreatic dissection. ${ }^{17}$ The details of these three steps are shown in our previous reports. 17,26

Regarding splenic hilar nodal dissection, we previously reported that the initial mobilization of the pancreatic body and downward retraction of the pancreas by gauze traction to obtain an extensive surgical field at the upper border of the pancreas was the keys to success. ${ }^{27}$ Recently, we have found that the outermost layeroriented approach could be nicely applied to the splenic hilar

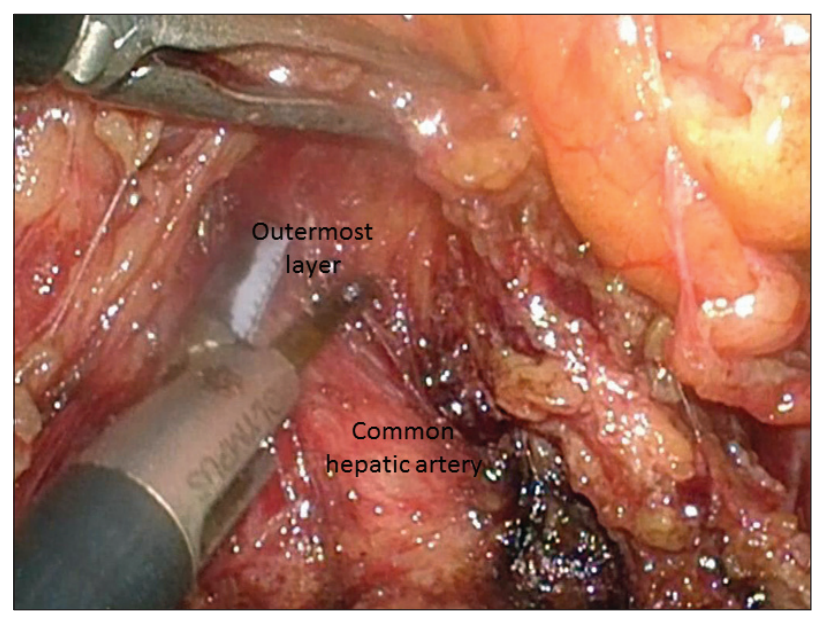

Fig. 2. Outermost layer between the autonomic nerve sheath of the common hepatic artery and adipose tissue bearing \#8a lymph nodes. area; this approach was used for spleen-preserving D2 dissection as well as for D2 dissection combined with splenectomy or pancreaticosplenectomy (PS) in laparoscopic total gastrectomy (LTG) (unpublished data).

\section{4) Intracorporeal anastomosis}

To fully utilize the advantages of LG, totally LG with intracorporeal anastomosis is promising. We have preferred to perform intracorporeal anastomosis with linear staplers. In distal gastrectomy, we have been using delta-shaped anastomosis for Billroth-I reconstruction, ${ }^{28}$ antiperistaltic side-to-side anastomosis for Billroth-II reconstruction, and functional end to end anastomosis for Roux-en-Y reconstruction. In total gastrectomy, we have been using functional end to end anastomosis ${ }^{25}$ and the overlap method ${ }^{29}$ for intraabdominal and intrathoracic esophagojejunostomy, respectively. In proximal gastrectomy, the modified overlap method with no-knife stapler has been used. ${ }^{30}$ The details of intracorporeal anastomosis in LG are well summarized in a recently published review by Hosogi and Kanaya. $^{30}$

\section{5) Outcomes}

Short-term and long-term outcomes of LG for AGC at our institute have been satisfactory from both technical and oncological points of view (LG vs. OG: morbidity, $1.1 \%$ vs. $0 \%, \mathrm{P}=0.519$; mortality, $24.2 \%$ vs. $28.5 \%, \mathrm{P}=0.402 ; 5$-year disease free survival, $65.8 \%$ vs. $62.0 \%, \mathrm{P}=0.737$; overall survival, $68.1 \%$ vs. $63.7 \%$, $\mathrm{P}=0.968)$. Details are demonstrated in our previous report. ${ }^{7}$

\section{Current status of LG for AGC}

Since the 1st report of LG in 1994 by Kitano, much of our knowledge on the feasibility, safety, and benefits of LG for gastric cancer has been derived from studies conducted in East Asia, largely because Japan and Korea have the highest gastric cancer rates in the world. ${ }^{1,431,32}$ LG has recently been accepted for EGC worldwide, and a laparoscopic approach is employed in approximately $20 \%$ of gastric cancer surgeries in Japan. ${ }^{4,5,11}$ However, this approach, even for EGC, has still been recognized as investigational treatment but not as a standard procedure in daily practice because the benefits of this potentially minimally invasive procedure have only been shown by small comparative studies, and its effectiveness in EGC has currently been assessed in RCTs in Japan and Korea (JCOG0912 and Korean Laparoscopic Gastrointestinal Surgery Study 01 trials). ${ }^{3,11,32}$ It is needless to say that the rationale for its application particularly to AGC has yet to 
Uyama l, et al

be determined at present. ${ }^{32}$ So far, outcomes of LG related to AGC treatment have been reported as shown below.

\section{1) $L G$ with $D 2$ dissection}

There has been only one meta-analysis reporting on LG with D2 dissection. ${ }^{5}$ Although this report was based mostly on case control studies, LG with D2 dissection resulted in approximately a longer duration of operation by one hour, reduced blood loss, less total postoperative complications, less pain, faster bowel function recovery, and shorter hospital stay, with a similar number of harvested lymph nodes as well as a similar overall survival rate in comparison with OG with D2 dissection, as demonstrated in our previous report. ${ }^{5,7}$ Regarding postoperative complications, LG with D2 dissection reduced wound infection and postoperative ileus, whereas duodenal stump leakage and anastomotic leakage were no different between LG and OG. ${ }^{5}$

\section{2) Distal gastrectomy}

Although the majority of patients had stage I or II cancer with an average follow-up period of 38 months or less in recent studies on LDG for AGC, Gordon et al. ${ }^{33}$ demonstrated that LDG with D2 dissection for AGC was feasible and could reproduce the survival rate equivalent to that for open distal gastrectomy (ODG) using a cohort in which $67.2 \%$ had a tumor of stage IIB or higher with an average follow-up period of 49.2 months. The short-term benefits of LDG shown in this study were consistent with those of the current studies. ${ }^{33}$ More evidence-based large prospective clinical trials would be required to validate the use of $\mathrm{LDG}$ for $\mathrm{AGC}^{4}$

\section{3) Total gastrectomy}

Since our 1st report in 1999 on LTG in patients with proximal gastric cancer, there have been only two reports on LTG in a relatively large series of patients with AGC. ${ }^{7,25,34}$ These two studies concluded that LTG with D2 dissection for AGC is technically feasible and safe when performed by experienced laparoscopic surgeons, and long-term follow-up is mandatory to validate oncological outcome. ${ }^{7,34}$ According to our database of over 1,000 patients who underwent LG, LTG but not LDG was determined as one of the independent risk factors for postoperative complications (unpublished data), suggesting that LTG is more technically demanding than $\mathrm{LDG}$.

\section{4) Learning curve}

The learning curve for LG for gastric cancer is steep. It has been reported that at least 50 to 60 cases are necessary to ensure an optimal operative performance of LDG for EGC, suggesting that more cases should be required to reach a plateau in the learning curve for LG with D2 dissection for AGC. ${ }^{4}$

\section{Future directions}

There have been several novel arising technologies which could further improve the precision, meticulousness and reproducibility of LG for AGC.

\section{1) Robotic approach: da Vinci Surgical System}

da Vinci Surgical System (Intuitive Surgical, Inc., Sunnyvale, CA, USA) is characterized by a three-dimensional, ten-fold magnified vivid view of the operating field, instruments with articulating endeffectors and 7 Degrees of Freedom, tremor filtering, and motion scaling. ${ }^{17,35}$ These characteristics may help surgeons overcome the limitations of conventional LG. In fact, there have been a number of clinical studies demonstrating that the use of a surgical robot in LG was associated with a longer operation time, reduced blood loss, and shorter hospital stay; however, the economic feasibility of robotic devices for gastrectomy remains an important and unanswered issue. ${ }^{4,1736}$ Since the introduction of da Vinci S HD Surgical System (Intuitive Surgical, Inc.) in 2009, we have performed more than 130 robotic gastrectomies. According to our database, there are almost no significant differences in short-term outcomes irrespective of the extent of lymph node dissection (robotic distal gastrectomy with D1+ vs. robotic distal gastrectomy with D2) and the addition of extended multi-organ resection (robotic total gastrectomy alone vs. robotic total gastrectomy with PS). Moreover, the use of a surgical robot significantly reduced postoperative local complications in LTG (unpublished data). These data suggest that the use of a surgical robot may be more beneficial for surgeries that require advanced skills rather than those that require basic or intermediate skills. Nevertheless, there are several issues to be solved such as long operation time, high cost, and limited experience and evidence.

\section{2) Integrated Bipolar and Ultrasonic Energy Platform (THUNDERBEAT)}

Advanced laparoscopic procedures are largely dependent on either mechanical methods of hemostasis or on energy-based surgical devices. THUNDERBEAT (Fig. 3, Olympus Medical Systems Corp, Tokyo, Japan) are novel surgical scissors that simultaneously deliver ultrasonically generated frictional heat 


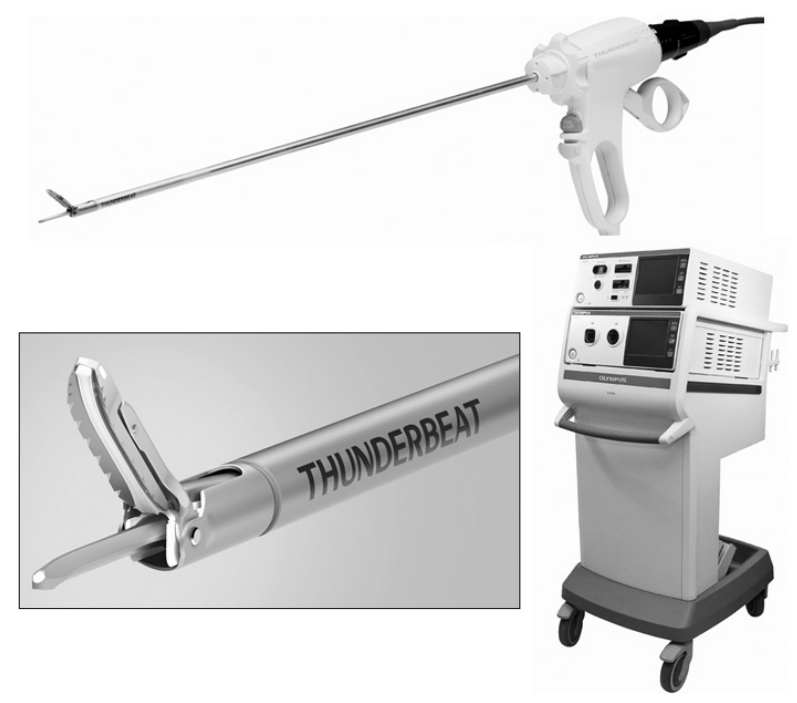

Fig. 3. THUNDERBEAT.

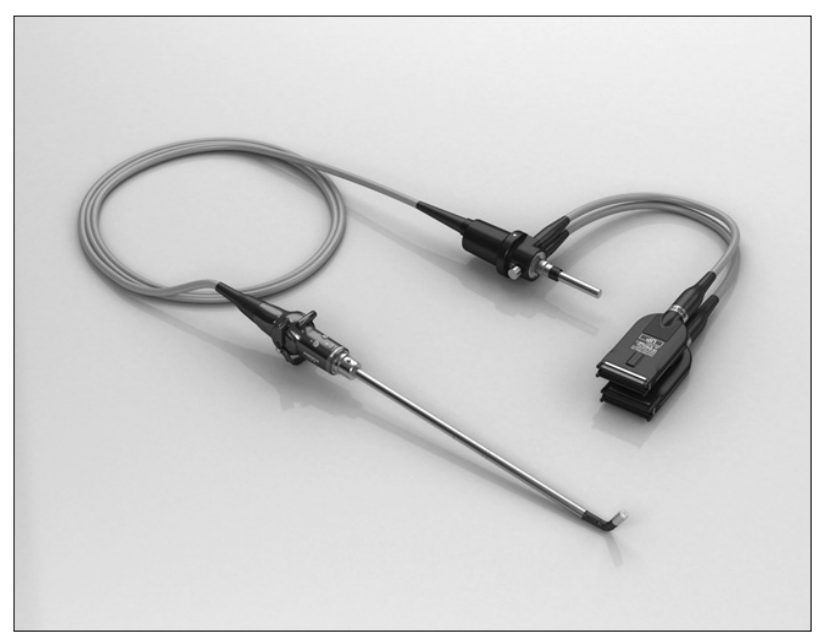

Fig. 4. ENDOEYE FLEX 3D DEFLECTABLE VIDEOSCOPE.

energy and bipolar heat energy and have the potential to surpass the dissection speed of ultrasonic devices with the sealing efficacy of bipolar clamps. ${ }^{37}$ THUNDERBEAT may further improve the technical aspects of LG, and lead to reduced operative time and blood loss.

\section{3) 3D imaging system}

The lack of stereoscopic vision is one of the major factors that make the laparoscopic procedure more difficult and stressful. ${ }^{38}$ The up-coming 3D imaging system with ENDOEYE FLEX 3D DEFLECTABLE VIDEOSCOPE (Fig. 4, Olympus Medical Systems Corp) may increase the accuracy of laparoscopy performance, with greater depth perception and only minimal dizziness.

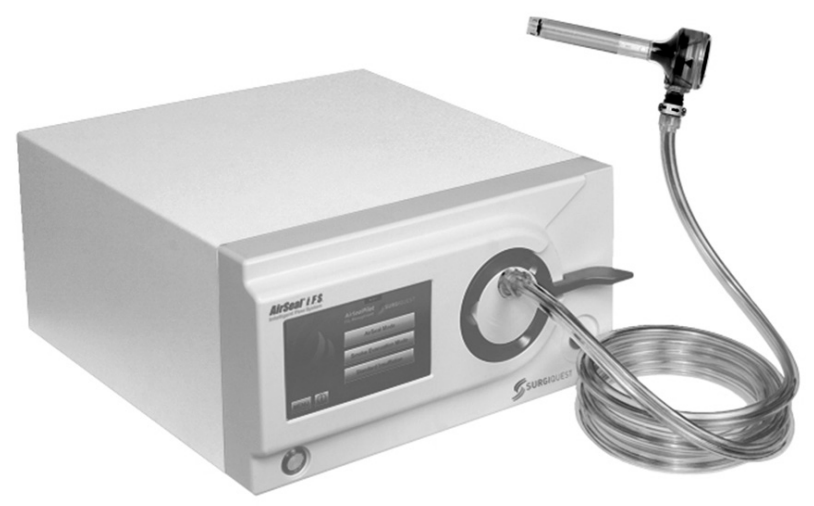

Fig. 5. AirSeal System.

\section{4) Intelligent and integrated access system for laparo- scopic and robotic surgery: AirSeal System}

In $\mathrm{LG}$, especially for AGC, stable pneumoperitoneum is very important. The AirSeal System (Fig. 5, SurgiQuest Inc., Milford, $\mathrm{CT}$, USA) is a novel laparoscopic $\mathrm{CO}_{2}$ insufflation system composed of the Intelligent Flow System Air Seal control, the AirSeal valve-less Trocar and the Air Seal Mode Evacuation Tri-lumen Filter Tube Set. ${ }^{39}$ This system creates closed loop $\mathrm{CO}_{2}$ filtration and allows rapid inflow to maintain a stable intraabdominal pressure even during constant aspiration or a sudden gas loss. Thus, the use of AirSeal has been reported to be associated with decreased camera smudging, better vision due to constant smoke evacuation, and a more stable pneumoperitoneum. ${ }^{40}$

\section{Conclusions}

It has been clearly shown that LG has considerable short-term benefits over OG. Although further investigation would be required to demonstrate the oncological safety of LG for AGC, we expect that the indications for a minimally invasive approach will continue to expand with ongoing improvements in technology.

\section{References}

1. Crew KD, Neugut AI. Epidemiology of gastric cancer. World J Gastroenterol 2006;12:354-362.

2. Sano T, Sasako M, Yamamoto S, Nashimoto A, Kurita A, Hiratsuka $M$, et al. Gastric cancer surgery: morbidity and mortality results from a prospective randomized controlled trial comparing D2 and extended para-aortic lymphadenectomy-Japan Clinical Oncology Group study 9501. J Clin Oncol 2004;22:2767-2773. 
Uyama I, et al

3. NCCN Guidelines Version 2.2011 Gastric Cancer. http://www. nccn.org/professionals/physician_gls/f_guidelines.asp

4. Bamboat ZM, Strong VE. Minimally invasive surgery for gastric cancer. J Surg Oncol 2013;107:271-276.

5. Wei HB, Wei B, Qi CL, Chen TF, Huang Y, Zheng ZH, et al. Laparoscopic versus open gastrectomy with D2 lymph node dissection for gastric cancer: a meta-analysis. Surg Laparosc Endosc Percutan Tech 2011;21:383-390.

6. Angst E, Hiatt JR, Gloor B, Reber HA, Hines OJ. Laparoscopic surgery for cancer: a systematic review and a way forward. J Am Coll Surg 2010;211:412-423.

7. Shinohara T, Satoh S, Kanaya S, Ishida Y, Taniguchi K, Isogaki J, et al. Laparoscopic versus open D2 gastrectomy for advanced gastric cancer: a retrospective cohort study. Surg Endosc 2013;27:286-294.

8. Fujii M, Sasaki J, Nakajima T. State of the art in the treatment of gastric cancer: from the 71st Japanese Gastric Cancer Congress. Gastric Cancer 1999;2:151-157.

9. Sano T, Aiko T. New Japanese classifications and treatment guidelines for gastric cancer: revision concepts and major revised points. Gastric Cancer 2011;14:97-100.

10. Sasako M, Sano T, Yamamoto S, Kurokawa Y, Nashimoto A, Kurita A, et al; Japan Clinical Oncology Group. D2 lymphadenectomy alone or with para-aortic nodal dissection for gastric cancer. N Engl J Med 2008;359:453-462.

11. Japanese Gastric Cancer Association. Japanese gastric cancer treatment guidelines 2010 (ver. 3). Gastric Cancer 2011;14:113123.

12. Hartgrink HH, van de Velde CJ, Putter H, Bonenkamp JJ, Klein Kranenbarg E, Songun I, et al. Extended lymph node dissection for gastric cancer: who may benefit? Final results of the randomized Dutch gastric cancer group trial. J Clin Oncol 2004;22:2069-2077.

13. Songun I, Putter H, Kranenbarg EM, Sasako M, van de Velde CJ. Surgical treatment of gastric cancer: 15-year follow-up results of the randomised nationwide Dutch D1D2 trial. Lancet Oncol 2010;11:439-449.

14. Cuschieri A, Weeden S, Fielding J, Bancewicz J, Craven J, Joypaul V, et al. Patient survival after D1 and D2 resections for gastric cancer: long-term results of the MRC randomized surgical trial. Surgical Co-operative Group. Br J Cancer 1999;79:1522-1530.

15. Kimura T, Kimura K, Suzuki K, Sakai S, Ohtomo Y, Sakuramachi S, et al. Laparoscopic cholecystectomy: the Japanese experi- ence. Surg Laparosc Endosc 1993;3:194-198.

16. Koeda K, Nishizuka S, Wakabayashi G. Minimally invasive surgery for gastric cancer: the future standard of care. World J Surg 2011;35:1469-1477.

17. Uyama I, Kanaya S, Ishida Y, Inaba K, Suda K, Satoh S. Novel integrated robotic approach for suprapancreatic D2 nodal dissection for treating gastric cancer: technique and initial experience. World J Surg 2012;36:331-337.

18. Uyama I, Ogiwara H, Takahara T, Kato Y, Kikuchi K, Iida S. Laparoscopic Billroth I gastrectomy for gastric ulcer: technique and case report. Surg Laparosc Endosc 1995;5:209-213.

19. Uyama I, Ogiwara H, Takahara T, Furuta T, Kikuchi K, Iida S. Laparoscopic minilaparotomy Billroth I gastrectomy with extraperigastric lymphadenectomy for early gastric cancer using an abdominal wall-lifting method. J Laparoendosc Surg 1995;5:181-187.

20. Uyama I, Ogiwara H, Takahara T, Kikuchi K, Iida S. Laparoscopic and minilaparotomy proximal gastrectomy and esophagogastrostomy: technique and case report. Surg Laparosc Endosc 1995;5:487-491.

21. Uyama I, Ogiwara H, Iida S, Takahara T, Furuta T, Kikuchi K. Laparoscopic minilaparotomy pancreaticoduodenectomy with lymphadenectomy using an abdominal wall-lift method. Surg Laparosc Endosc 1996;6:405-410.

22. Uyama I, Ogiwara H, Takahara T, Kikuchi K, Iida S. Gasless laparoscopic and minilaparotomy Billroth I gastrectomy with hepatectomy for advanced gastric cancer with liver metastasis : technique and case report. Min Invas Ther \& Allied Technol 1996;5:281-285.

23. Uyama I, Ogiwara H, Takahara T, Kikuchi K, Iida S, Kubota $\mathrm{T}$, et al. Spleen- and pancreas-preserving total gastrectomy with superextended lymphadenectomy including dissection of the para-aortic lymph nodes for gastric cancer. J Surg Oncol 1996;63:268-270.

24. Uyama I, Sugioka A, Matsui H, Fujita J, Komori Y, Hasumi A. Laparoscopic D2 lymph node dissection for advanced gastric cancer located in the middle or lower third portion of the stomach. Gastric Cancer 2000;3:50-55.

25. Uyama I, Sugioka A, Fujita J, Komori Y, Matsui H, Hasumi A. Laparoscopic total gastrectomy with distal pancreatosplenectomy and D2 lymphadenectomy for advanced gastric cancer. Gastric Cancer 1999;2:230-234.

26. Kanaya S, Haruta S, Kawamura Y, Yoshimura F, Inaba K, Hiramatsu Y, et al. Video: laparoscopy distinctive technique for 
Laparoscopic Radical Gastrectomy

suprapancreatic lymph node dissection: medial approach for laparoscopic gastric cancer surgery. Surg Endosc 2011;25:39283929.

27. Shinohara T, Kanaya S, Taniguchi K, Fujita T, Yanaga K, Uyama I. Laparoscopic total gastrectomy with D2 lymph node dissection for gastric cancer. Arch Surg 2009;144:1138-1142.

28. Kanaya S, Gomi T, Momoi H, Tamaki N, Isobe H, Katayama T, et al. Delta-shaped anastomosis in totally laparoscopic Billroth I gastrectomy: new technique of intraabdominal gastroduodenostomy. J Am Coll Surg 2002;195:284-287.

29. Inaba K, Satoh S, Ishida Y, Taniguchi K, Isogaki J, Kanaya S, et al. Overlap method: novel intracorporeal esophagojejunostomy after laparoscopic total gastrectomy. J Am Coll Surg 2010;211:e25-29.

30. Hosogi H, Kanaya S. Intracorporeal anastomosis in laparoscopic gastric cancer surgery. J Gastric Cancer 2012;12:133139.

31. Kitano S, Iso Y, Moriyama M, Sugimachi K. Laparoscopy-assisted Billroth I gastrectomy. Surg Laparosc Endosc 1994;4:146148.

32. Kodera Y, Fujiwara M, Ohashi N, Nakayama G, Koike M, Morita S, et al. Laparoscopic surgery for gastric cancer: a collective review with meta-analysis of randomized trials. J Am Coll Surg 2010;211:677-686.

33. Gordon AC, Kojima K, Inokuchi M, Kato K, Sugihara K. Longterm comparison of laparoscopy-assisted distal gastrectomy and open distal gastrectomy in advanced gastric cancer. Surg Endosc 2013;27:462-470.
34. Lee JH, Ahn SH, Park do J, Kim HH, Lee HJ, Yang HK. Laparoscopic total gastrectomy with D2 lymphadenectomy for advanced gastric cancer. World J Surg 2012;36:2394-2399.

35. Suda K, Ishida Y, Kawamura Y, Inaba K, Kanaya S, Teramukai $S$, et al. Robot-assisted thoracoscopic lymphadenectomy along the left recurrent laryngeal nerve for esophageal squamous cell carcinoma in the prone position: technical report and shortterm outcomes. World J Surg 2012;36:1608-1616.

36. Isogaki J, Haruta S, Man-I M, Suda K, Kawamura Y, Yoshimura F, et al. Robot-assisted surgery for gastric cancer: experience at our institute. Pathobiology 2011;78:328-333.

37. Seehofer D, Mogl M, Boas-Knoop S, Unger J, Schirmeier A, Chopra S, et al. Safety and efficacy of new integrated bipolar and ultrasonic scissors compared to conventional laparoscopic 5-mm sealing and cutting instruments. Surg Endosc 2012;26:2541-2549.

38. Kong SH, Oh BM, Yoon H, Ahn HS, Lee HJ, Chung SG, et al. Comparison of two- and three-dimensional camera systems in laparoscopic performance: a novel 3D system with one camera. Surg Endosc 2010;24:1132-1143.

39. Horstmann M, Horton K, Kurz M, Padevit C, John H. Prospective comparison between the airSeal $(\mathrm{R})$ system valve-less trocar and a standard versaport ${ }^{\mathrm{TM}}$ plus $\mathrm{V} 2$ trocar in roboticassisted radical prostatectomy. J Endourol 2013. [Epub ahead of print]

40. Herati AS, Atalla MA, Rais-Bahrami S, Andonian S, Vira MA, Kavoussi LR. A new valve-less trocar for urologic laparoscopy: initial evaluation. J Endourol 2009;23:1535-1539. 